\title{
Lactobacillus reuteri LR1 Improved Expression of Genes of Tight Junction Proteins via the MLCK Pathway in IPEC-1 Cells during Infection with Enterotoxigenic Escherichia coli K88
}

\author{
Hongbo Yi $\mathbb{D}$, Li Wang $(\mathbb{D}$, Yunxia Xiong, Zhilin Wang, Yueqin Qiu, Xiaolu Wen, \\ Zongyong Jiang $\mathbb{\oplus}$, Xuefen Yang, and Xianyong Ma \\ State Key Laboratory of Livestock and Poultry Breeding, Ministry of Agriculture Key Laboratory of Animal Nutrition and Feed \\ Science in South China, Guangdong Public Laboratory of Animal Breeding and Nutrition, Guangdong Key Laboratory of Animal \\ Breeding and Nutrition, Institute of Animal Science, Guangdong Academy of Agricultural Sciences, 1 Dafeng 1st Street, \\ Guangzhou 510640, China
}

Correspondence should be addressed to Li Wang; wangli1@gdaas.cn and Zongyong Jiang; jiangz28@qq.com

Received 4 February 2018; Revised 26 June 2018; Accepted 9 July 2018; Published 19 August 2018

Academic Editor: Elisabetta Buommino

Copyright (c) 2018 Hongbo Yi et al. This is an open access article distributed under the Creative Commons Attribution License, which permits unrestricted use, distribution, and reproduction in any medium, provided the original work is properly cited.

\begin{abstract}
Intestinal epithelial barrier damage disrupts immune homeostasis and leads to many intestinal disorders. Lactobacillus reuteri strains have probiotic functions in their modulation of the microbiota and immune system in intestines. In this study, the effects of $L$. reuteri LR1, a new strain isolated from the feces of weaning piglets, on intestinal epithelial barrier damage in IPEC-1 cells caused by challenge with enterotoxigenic Escherichia coli (ETEC) K88 were examined. It was found that L. reuteri LR1, in large part, offset the ETEC K88-induced increase in permeability of IPEC-1 cell monolayers and decreased the adhesion and invasion of the coliform in IPEC-1 cells. In addition, L. reuteri LR1 increased transcript abundance and protein contents of tight junction (TJ) proteins zonula occluden-1 (ZO-1) and occludin in ETEC K88-infected IPEC-1 cells, whereas it had no effects on claudin-1 and F-actin expression. Using colloidal gold immunoelectron microscopy, these effects of L. reuteri LR1 on ZO-1 and occludin content in IPEC-1 cells were confirmed. By using ML-7, a selective inhibitor of myosin light-chain kinase (MLCK), the beneficial effect of $L$. reuteri LR1 on contents of ZO-1 and occludin was shown to be dependent on the MLCK pathway. In conclusion, $L$. reuteri LR1 had beneficial effects on epithelial barrier function consistent with increasing ZO-1 and occludin expression via a MLCK-dependent manner in IPEC-1 cells during challenge with ETEC K88.
\end{abstract}

\section{Introduction}

The intestinal epithelial barrier plays an essential role in the host defense against pathogen infection [1]. An impaired epithelial barrier disrupts immune homeostasis and exacerbates inflammation in many diseases, such as postweaning diarrhea stress, enteric pathogen infection, inflammatory bowel disease (IBD), irritable bowel syndrome, obesity, metabolic syndrome, and liver diseases [2-6]. The tight junctions (TJ) between adjacent epithelial cells create a semipermeable barrier that prevents bacteria and other harmful substances from crossing the epithelium [7]. Disruptions of TJ proteins increase the permeability of the epithelial barrier and cause inflammation in the intestine [8], leading to many intestinal diseases. Although antibiotics have been widely used to treat intestinal diseases in past decades, recent studies have demonstrated that antibiotic exposure disrupts both the normal composition of intestinal microbiota and expression of TJ proteins hence damaging intestinal epithelial barrier function [9-11]. All this emphasizes the need to identify safe and effective agents for the treatment of intestinal diseases associated with damage to the epithelial barrier.

Lactobacillus reuteri (L. reuteri) strains are important members of the commensal microbiota in intestines of humans and other animals and have been demonstrated to regulate the development of the immune system in the gut $[12-14]$. L. reuteri can produce reuterin, which exhibits a broad-spectrum antimicrobial activity against intestinal 
pathogens [15-17]. In addition, human $L$. reuteri reduces intestinal inflammation by inhibiting the toll-like receptor 4- (TLR4-) nuclear factor $\kappa \mathrm{B}$ (NF- $\kappa \mathrm{B})$ signaling pathway $[18,19]$. It also improved intestinal epithelial barrier function by promoting cell migration [20]. Previous studies have demonstrated that some probiotics increase the expression of intestinal tight junction proteins through p38/MAPK or myosin light-chain kinase (MLCK) signaling pathways [21]. The latter regulates myosin light-chain (MLC) phosphorylation and then modulates intercellular permeability and apoptosis of intestinal epithelial cells [22, 23]. The strain $L$. reuteri LR1 was isolated from the feces of healthy weaned piglets in our previous study, and its 16S rRNA sequence had been deposited in the GenBank database (accession number KT205306) [24] The L. reuteri LR1 showed beneficial effects on intestinal epithelial barrier functions [24]. The effects and underlying mechanisms of L. reuteri LR1 on intestinal epithelial barrier function during challenge with enterotoxigenic Escherichia coli (ETEC) are, as yet, incomplete.

The objective of this study was to investigate effects and underlying mechanism of $L$. reuteri LR1 on ETEC K88-induced damage of the epithelial barrier function in an in vitro model using intestinal porcine epithelial cells.

\section{Materials and Methods}

2.1. Bacterial Cultures. L. reuteri LR1 was isolated from the feces of a healthy weaned piglet (Duroc $\times$ Landrace $\times$ Large White), as described [24]. L. reuteri $\mathrm{LR} 1$ was grown at $37^{\circ} \mathrm{C}$ for $18 \mathrm{~h}$ in MRS broth. ETEC K88 was obtained from the Institute of Veterinary Drug Control of China and grown in lysogeny broth at $37^{\circ} \mathrm{C}$ for $16 \mathrm{~h}$. Bacterial cells of ETEC K88 and $L$. reuteri LR1 were suspended at the required concentration in Dulbecco's Modified Eagle's Medium (DMEM, Invitrogen, Carlsbad, CA).

2.2. Cell Culture. Intestinal porcine epithelial cells (IPEC-1) were a gift from Dr. Guoyao Wu (Texas A\&M University). The cells were cultured in DMEM supplemented with 10\% inactivated fetal bovine serum (Gibco) and antibiotics $(100 \mathrm{U} / \mathrm{ml}$ penicillin and $100 \mu \mathrm{g} / \mathrm{ml}$ streptomycin sulfate) at $37^{\circ} \mathrm{C}$ under $5 \% \mathrm{CO}_{2}$ in a humidified incubator.

IPEC-1 cells were cultured in Transwell dishes (Corning Life Sciences, Corning, NY) for $21 \mathrm{~d}$ as before [24]. Monolayers of IPEC- 1 cells were incubated for $6 \mathrm{~h}$ in DMEM without serum or antibiotics, in the presence of ETEC K88 $\left(1 \times 10^{7} \mathrm{CFU}\right)$, L. reuteri LR1 $\left(1 \times 10^{8} \mathrm{CFU}\right)$, or both, in the upper chamber. Permeability of the IPEC- 1 cell monolayers was measured with FITC-dextran (4400 Da, Sigma-Aldrich, St Louis, MO) [24]. IPEC-1 cells were collected for enumeration of ETEC K88, real-time PCR (qPCR), and Western blotting analysis. Six wells per treatment were used, and the results were representative of 3 independent experiments.

2.3. Treatment with Inhibitor $M L-7$. IPEC-1 cells were seeded in 6 -well plates $\left(5 \times 10^{5}\right.$ cells per well) and cultured for $24 \mathrm{~h}$. The cells were pretreated with $50 \mu \mathrm{M}$ ML-7 (a selective inhibitor of MLCK) or vehicle (0.1\% DMSO) for $6 \mathrm{~h}$ and then treated with $L$. reuteri LR1 $\left(1 \times 10^{8} \mathrm{CFU}\right.$ for $\left.6 \mathrm{~h}\right)$ before exposure to ETEC K88 $\left(1 \times 10^{7} \mathrm{CFU}\right.$ for $\left.1 \mathrm{~h}\right)$, then IPEC-1 cells were collected for Western blotting analysis. Six wells per treatment were used.

2.4. Colloidal Gold Immunoelectron Microscopy. After incubating for $6 \mathrm{~h}$ with medium, ETEC K88, or ETEC K88 plus $L$. reuteri LR1, as above, in the upper chamber of Transwell dishes, monolayers of IPEC- 1 cells were fixed with $2.5 \%$ glutaraldehyde for $30 \mathrm{~min}$ and then dehydrated in a graded series of ethanol $(30 \%, 50 \%$, and $70 \%)$. The cells were transferred into epoxy resin Epon812 overnight and then heated for $72 \mathrm{~h}$ (each step of $35^{\circ} \mathrm{C}, 45^{\circ} \mathrm{C}$, and $60^{\circ} \mathrm{C}$ for $24 \mathrm{~h}$ ). Specimens were sectioned with a LKB-V ultramicrotome (LKB Bromma) and put on a nickel screen. The sections were treated with $0.5 \mathrm{~mol} / 1 \mathrm{NH}_{4} \mathrm{Cl}$ for $15 \mathrm{~min}$ and then incubated in $3 \%$ hydrogen peroxide in the dark for $3 \mathrm{~min}$. After blocking for $30 \mathrm{~min}$ using $5 \% \mathrm{BSA}$, the sections were incubated with a primary antibody (1:20 dilution) against zonula occluden-1 (ZO-1) or occludin (Cell Signaling Technology, Danvers, MA) overnight. The sections were then incubated with a colloidal gold-labeled secondary antibody (1:50 dilution) for $1 \mathrm{~h}$. The sections were then stained with uranyl acetate and alkaline lead citrate for $15 \mathrm{~min}$ and visualized by transmission electron microscopy (Model H-7650, HITACHI, Japan).

2.5. Real-Time PCR. Total RNA was extracted using TRIpure reagent (Aidlab, Beijing, China). RNA quantity and quality were determined using a NanoDrop 1000 spectrophotometer (Thermo Fisher Scientific, Waltham, MA). The cDNA was generated using $1 \mu \mathrm{g}$ RNA with a PrimeScript RT reagent kit (Takara, Dalian, China). Real-time PCR was performed in triplicate using a CFX Connect detection system (BioRad, Hercules, CA). The primers used for real-time PCR are listed in Table 1. Each reaction included $10 \mu \mathrm{l}$ iTaq Universal SYBR Green Supermix (Bio-Rad), $0.8 \mu \mathrm{l}$ of each forward and reverse primers $(10 \mu \mathrm{M}), 2 \mu \mathrm{l} 10$-fold diluted

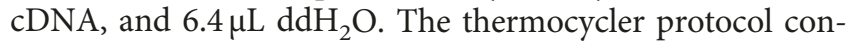
sisted of $10 \mathrm{~min}$ at $95^{\circ} \mathrm{C}$ and 40 cycles of $30 \mathrm{~s}$ at $95^{\circ} \mathrm{C}, 30 \mathrm{~s}$ at $60^{\circ} \mathrm{C}$, and $20 \mathrm{~s}$ at $72^{\circ} \mathrm{C}$. GAPDH and $\beta$-actin were used as housekeeping genes. The relative transcript abundance was calculated using the $2^{-\Delta \Delta \mathrm{Ct}}$ method, and the average of $\Delta \mathrm{Ct}$ $(\mathrm{GAPDH})$ and $\Delta \mathrm{Ct}(\beta$-actin) was used in this study. Treatment means were further normalized to the values obtained in the control treatment.

2.6. Western Blotting. Total protein was extracted from IPEC-1 cells using lysis buffer (KeyGEN, Nanjing, China) and clarified by centrifugation. Protein in supernatants was separated by $10 \%$ SDS-PAGE in a Bio-Rad system and transferred onto a polyvinylidene fluoride membrane (Bio-Rad). After blocking with 5\% BSA for $30 \mathrm{~min}$, the membranes were incubated overnight at $4^{\circ} \mathrm{C}$ with the appropriate primary antibodies for $\beta$-actin (Santa Cruz, CA), MLCK, phosphorylated MLCK, ZO-1, occludin, and F-actin (Cell Signaling Technology), followed by incubation with a horseradish peroxidase- (HRP-) conjugated secondary antibody for $1 \mathrm{~h}$. Bands were detected using Clarity Western ECL 
TABle 1: Primers used for real-time PCR in this study.

\begin{tabular}{lcr}
\hline Gene $^{1}$ & Primer sequence $\left(5^{\prime}-3^{\prime}\right)$ & Accession number \\
\hline \multirow{2}{*}{ ZO-1 } & Forward: AGCCCGAGGCGTGTTT & XM_013993251 \\
\multirow{2}{*}{ Occludin } & Reverse: GGTGGGAGGATGCTGTTG & \\
& Forward: GCACCCAGCAACGACAT & XM_005672525 \\
Claudin-1 & Feverse: CATAGACAGAATCCGAATCAC & NM_001244539 \\
& Reverse: GCACCTCATCATCTTCCAT & NM_001097454 \\
F-Actin & Forward: TCTGGAATGGTCGTTGGA & XM_003124280 \\
& Reverse: CCTTGAATGTGGTGTCTGA & Forward: CTGCGGCATCCACGAAACT \\
GAPDH & Reverse: AGGGCCGTGATCTCCTTCTG & NM_001206359 \\
\hline
\end{tabular}

${ }^{1}$ zonula occluden-1

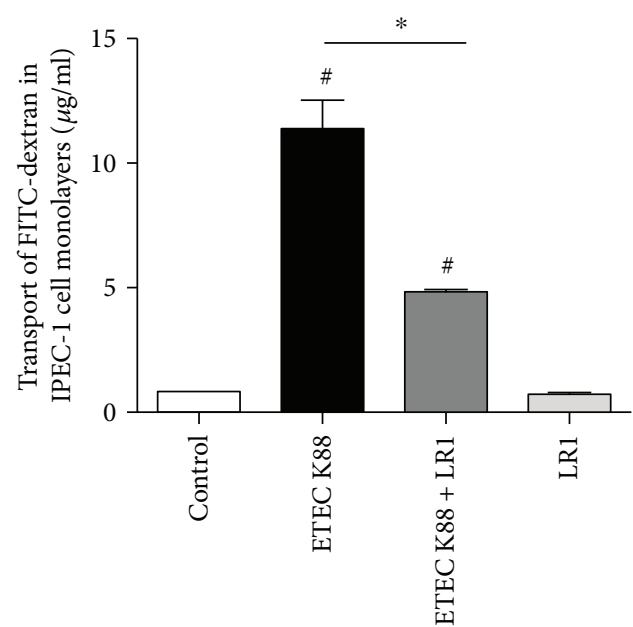

(a)

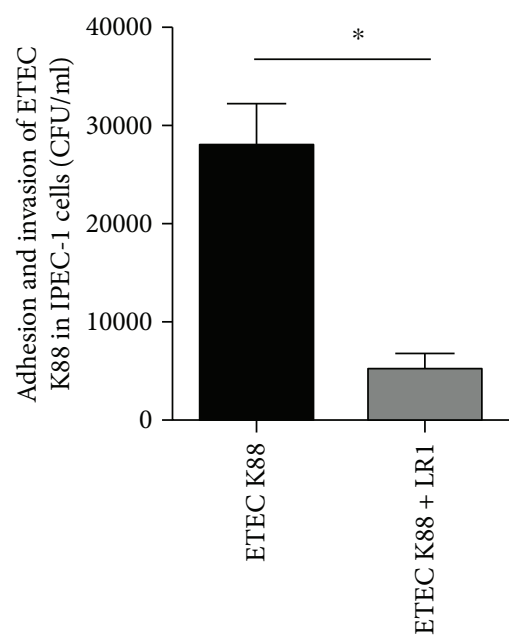

(b)

FIgURE 1: Permeability of IPEC-1 cell monolayers. (a) The transport of FITC-dextran across the IPEC-1 cell monolayers. (b) The adhesion and invasion of ETEC K88 in the IPEC-1 cell monolayers. All data are expressed as the mean \pm SEM $(n=6)$ and representative of 3 independent experiments. Differences were determined by one-way ANOVA. ${ }^{\#} P<0.05$ compared with control, ${ }^{*} P<0.05$.

Substrate (Bio-Rad). Band intensity was quantified using ImageJ software.

2.7. Statistical Analysis. Effects of treatment were assessed by one-way ANOVA using SPSS 16.0 software (SPSS Inc., Chicago, IL). Differences were considered to be significant at $P<0.05$.

\section{Results}

3.1. L. reuteri LR1 Attenuated ETEC K88-Induced Damages in the Epithelial Barrier of IPEC-1 Cells. The permeability of IPEC-1 cell monolayers was determined using FITCdextran. The transport of FITC-dextran in cells challenged with ETEC K88 was significantly increased when compared with the control (Figure 1(a)). Pretreatment with L. reuteri LR1 effectively decreased the transport of FITC-dextran in ETEC K88-infected IPEC-1 cell monolayers but had no effect when used alone. In addition, $L$. reuteri LR1 decreased the adhesion and invasion of ETEC K88 bacteria in the epithelial cells (Figure 1(b)). These results demonstrated that L. reuteri LR1 attenuated the ETEC K88-induced increase in the permeability of IPEC-1 cell monolayers.

3.2. L. reuteri LR1 Increased Gene Expression of TJ Proteins in ETEC K88-Infected IPEC-1 Cells. Challenge with ETEC K88 significantly decreased relative abundance of ZO-1 and occludin transcripts in IPEC-1 cells (Figures 2(a) and 2(b)). Pretreatment with $L$. reuteri LR1 again effectively offset this response to ETEC K88 resulting in much reduced decrease of ZO-1 and occludin expression. Neither ETEC K88 nor $L$. reuteri LR1 affected claudin transcripts in IPEC-1 cells (Figure 2(c)) while ETEC K88, but not L. reuteri LR1, decreased the expression of F-actin in IPEC-1 cells (Figure 2(d)). 
ZO-1

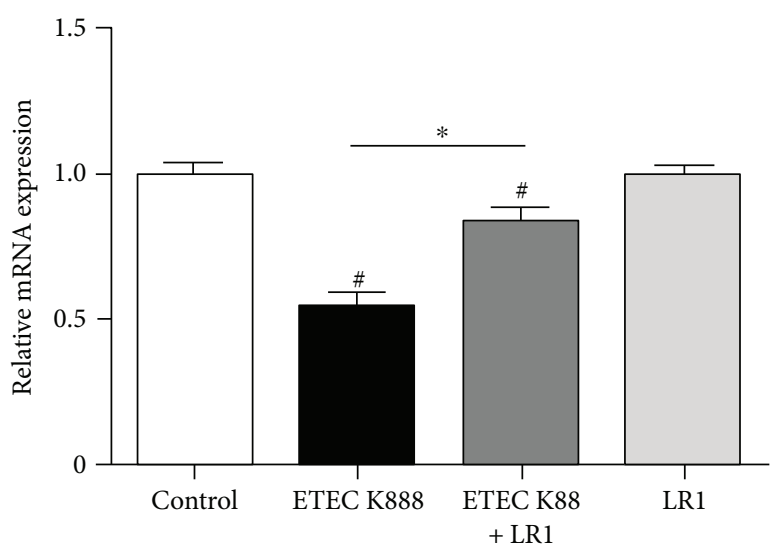

(a)

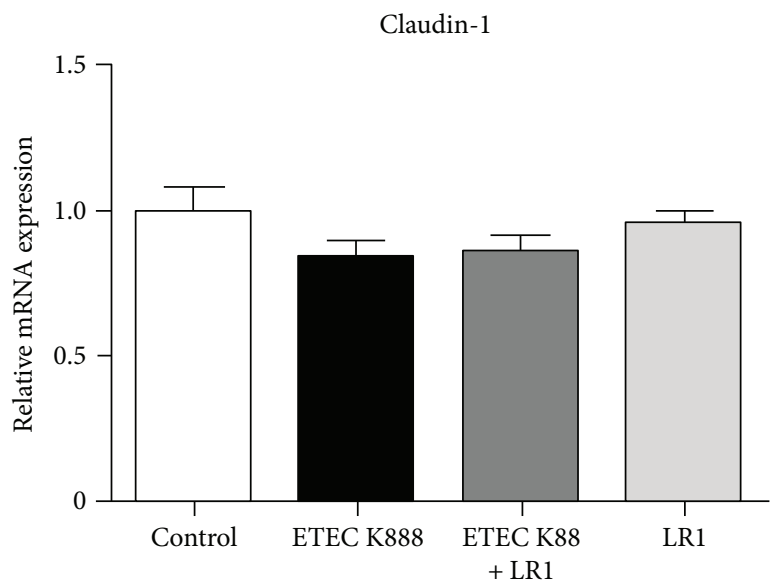

(c)

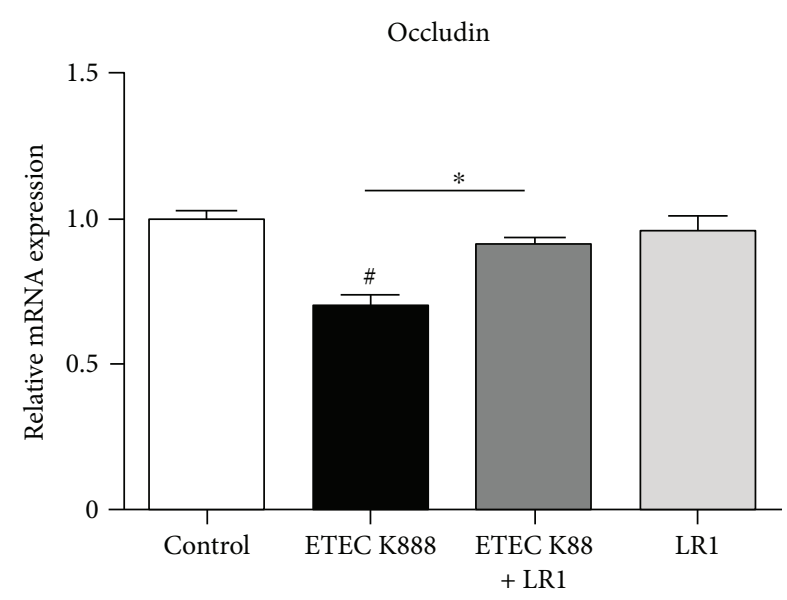

(b)

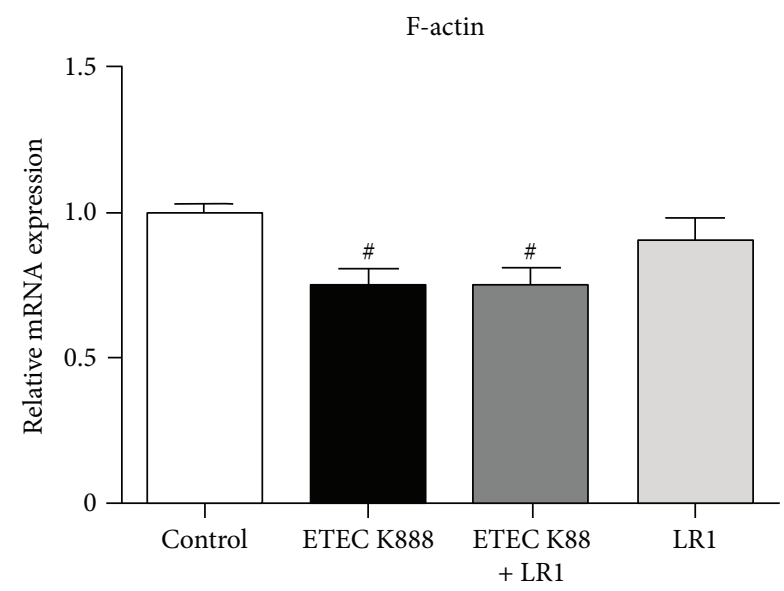

(d)

FIGURE 2: The mRNA levels of components of epithelial barrier in IPEC-1 cells. Relative transcript levels of ZO-1 (a), occludin (b), claudin-1 (c), and F-actin (d) in IPEC-1 cells were determined by qPCR. GAPDH and $\beta$-actin were used as housekeeping genes, and the $2^{-\Delta \Delta \mathrm{Ct}} \mathrm{method}$ was used to determine the relative abundance. Data were further normalized to values measured in control treatments. All data are expressed as the mean \pm SEM $(n=6)$ and representative of 3 independent experiments. Differences were determined by one-way ANOVA. ${ }^{\#} P<0.05$ compared with control, ${ }^{*} P<0.05$.

Western blotting was used to verify that the effects of L. reuteri LR1 during ETEC K88 challenge resulted in changes in protein content (Figure 3(a)). Consistent with the GPCR results, $L$. reuteri LR1 had no effect on the ETEC K88-induced decrease in F-actin protein, but it significantly increased ZO-1 and occludin protein content in ETEC K88-infected IPEC-1 cells (Figure 3(b)). These effects of $L$. reuteri LR1 on ZO-1 and occludin protein in ETEC-infected IPEC-1 cells were further confirmed by immunoultrastructural imaging (Figure 3(c)). Considered together, pretreatment with $L$. reuteri LR1 offset the gene expression and improved the status of the TJ proteins ZO-1 and occludin in IPEC-1 cells, otherwise challenged by ETEC K88.

3.3. LR1 Improved Expression of TJ Proteins in a MLCKDependent Manner. The effects of $L$. reuteri LR1 in influencing the expression of the genes encoding TJ proteins and the proteins themselves were examined at the level of MLCK and phosphorylated MLCK in ETEC K88-infected IPEC-1 cells. Challenge with ETEC K88 decreased the cellular contents of MLCK and phosphorylated MLCK proteins (Figure 4(a)). Although L. reuteri LR1 treatment alone had no effect on phosphorylation of MLCK in ETEC K88infected IPEC-1 cells, it diminished the extent of the decrease in MLCK protein caused by ETEC K88. The content of MLCK protein in IPEC-1 cells was suppressed by the selective inhibitor ML-7. It was then demonstrated that the increases in the content of ZO-1 and occludin caused by L. reuteri LR1 in ETEC K88-infected IPEC-1 cells were inhibited by ML-7. These data indicated that L. reuteri LR1 likely increased the expression of the TJ proteins via a MLCK-dependent pathway.

\section{Discussion}

Infection with the enterotoxigenic ETEC K88 usually impaired the integrity of the intestinal epithelial barrier $[25,26]$. Although the regulatory mechanism of beneficial probiotics on the intestinal epithelial barrier is still 


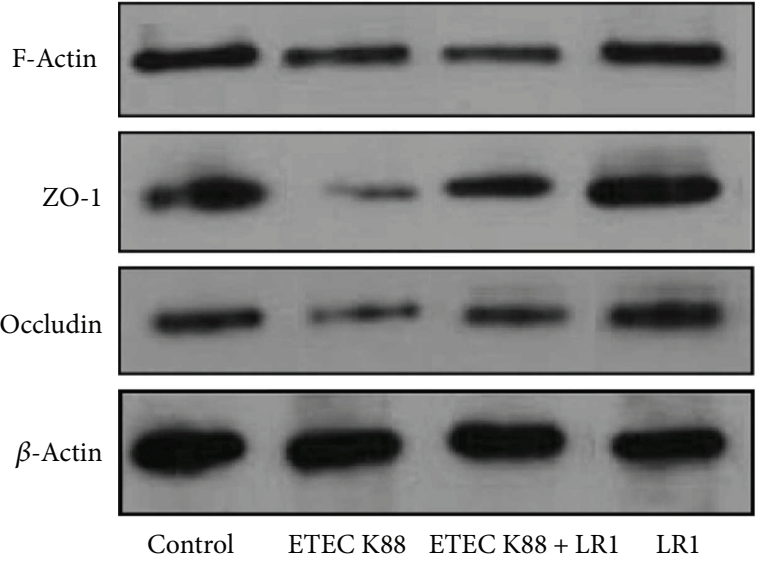

(a)
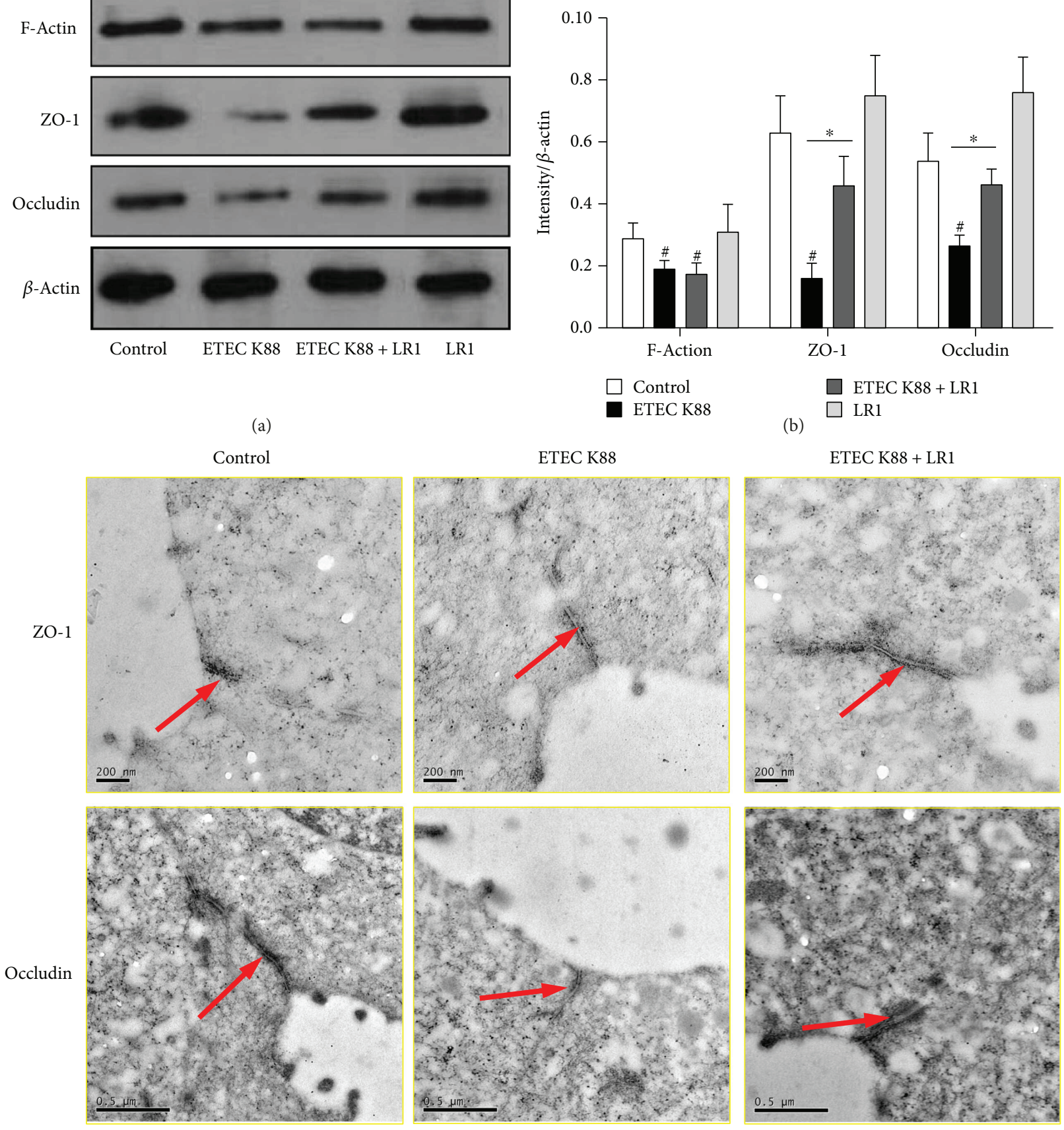

(c)

FIgURE 3: The contents of TJ proteins in IPEC-1 cells. (a) The contents of ZO-1, occludin, and F-actin in IPEC-1 cells were determined by Western blot analysis. (b) The relative intensity of target proteins to $\beta$-actin was calculated. All data are expressed as the mean \pm SEM $(n=3)$ and representative of 3 independent experiments. Differences were determined by one-way ANOVA. ${ }^{\#} P<0.05$ compared with control, ${ }^{*} P<0.05$. (c) ZO-1 and occludin in IPEC-1 cells visualized by colloidal gold immunoelectron microscopy.

unclear, it is established that some probiotics do improve barrier function, thereby maintaining intestinal homeostasis [27-30]. L. reuteri LR1, a new strain isolated from the feces of a healthy weaning piglet, exhibits both probiotic and functional properties in porcine intestinal epithelial cells [24]. It was therefore hypothesized that L. reuteri LR1 might protect against damage to epithelial barrier function in IPEC-1 cells, normally resulting from challenge with ETEC K88.

The present study clearly showed that $L$. reuteri LR1 substantially reduced the ETEC K88-induced increase in permeability of IPEC- 1 cell monolayers and reduced the adhesion and/or colonization by the coliform. In addition, $L$. reuteri $\mathrm{LR} 1$ offset the extent of decreases in transcripts 


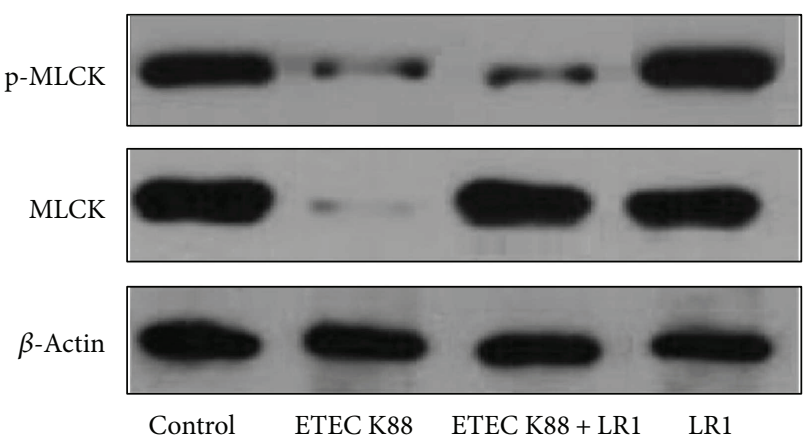

(a)

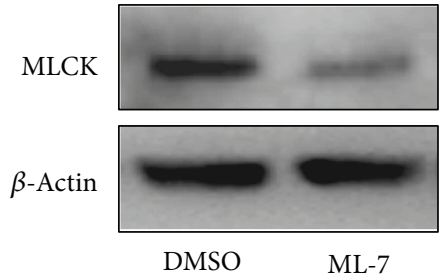

(b)
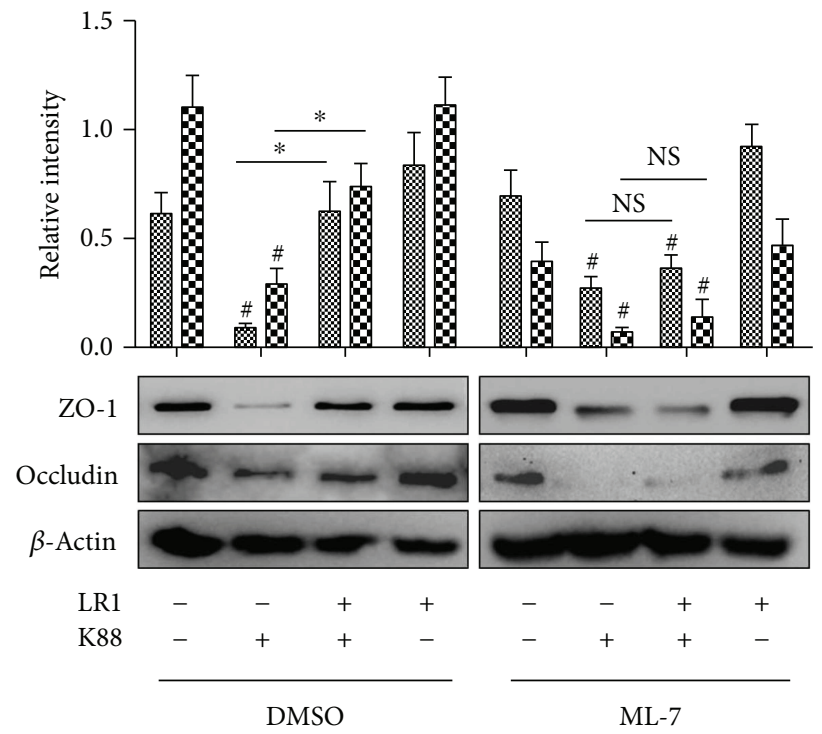

ZO- $1 / \beta$-actin

Q Occludin $/ \beta$-actin

(c)

FIGURE 4: L. reuteri LR1 improved content of TJ proteins in a MLCK-dependent manner. (a) Content of MLCK and phosphorylated MLCK as influenced by ETEC K88 with and without $L$. reuteri LR1 in IPEC-1 cells, by Western blotting. (b) Inhibiting effect of the selective inhibitor ML-7 on the action of L. reuteri LR1 on MLCK in IPEC-1 cells. (c) Content of ZO-1 and occludin in IPEC-1 cells with ML-7 treatment. All data are expressed as the mean $\pm \operatorname{SEM}(n=6)$ and representative of 3 independent experiments. Differences were determined by one-way ANOVA. ${ }^{\#} P<0.05$ compared with control, ${ }^{*} P<0.05$.

and proteins of TJ components ZO-1 and occludin provoked by ETEC K88 challenge in the IPEC-1 cells. Mechanistically, it was apparent that the protective effect of L. reuteri LR1 on ZO-1 and occludin was dependent upon the MLCK signaling pathway.

TJ proteins are the most important components of the intestinal epithelial barrier and play key roles in maintaining intestinal homeostasis by restricting the invasion of microbes and toxins $[1,22,31]$. The most important TJ proteins in the intestinal mucosa are ZO-1, occludin, and claudins [7], and the expression of all 3 in the intestine are decreased by infection with ETEC K88 [24, 30]. The present study showed that, for ZO-1 and occludin in IPEC-1 monolayers, this decrease from ETEC K88 infection was partially prevented by $L$. reuteri LR1. This finding is consistent with $L$. reuteri I5007 increasing expression of ZO-1 and occludin in similar intestinal porcine epithelial cells (IPEC-J2) [14]. Another probiotic, L. plantarum, ameliorated disruptions of ZO-1 and occludin in IPEC-J2 cells caused by ETEC K88 challenge [30]. Moreover, L. acidophilus and Streptococcus thermophilus attenuated decreases in ZO-1 expression in intestinal epithelial cells caused by enteroinvasive E.coli [32]. Similarly, L. reuteri 15007 increased claudin-1 expression in LPS-induced IPEC-J2 cells [14]. In contrast, in the present study, L. reuteri LR1 had no effect on claudin-1 expression in ETEC K88-infected IPEC-1 cells; perhaps the difference for claudin-1 might be due to a different strain of $L$. reuteri used. Nonetheless, the present study indicated that $L$. reuter $\mathrm{LR} 1$ improved TJ protein expression in IPEC-1 monolayer infected by ETEC K88, consistent with $L$. reuteri LR1 improving integrity of the intestinal epithelial barrier.

The regulatory mechanisms of TJ proteins are complex. Myosin light-chain (MLC) phosphorylation can lead to 
increased intercellular permeability, and MLCK plays the key role in the regulation of MLC phosphorylation. A previous study showed that MLCK can inhibit apoptosis of intestinal epithelial cells $[22,33]$. In the present study, ETEC K88 infection of IPEC-1 cells decreased protein levels of both MLCK and phosphorylated MLCK. Exposure to L. reuteri LR1 increased the levels of MLCK, but not those of phosphorylated MLCK, in ETEC-infected IPEC-1 cells. MLCK is known to regulate TJ barrier function [34]. The naphthalene sulfonamide ML-7 is a potent selective inhibitor of MLCK. A previous study in vascular endothelium showed that ML-7 could regulate TJ proteins ZO-1 and occludin expression through MLC phosphorylation and MLCK [35]. In the present study, ML-7 blocked the beneficial effect of L. reuteri LR1 on suppressed TJ proteins in IPEC-1 cells caused by ETEC K88, suggesting that $L$. reuteri LR1 likely acted through the MLCK signaling pathway.

Disruptions of TJ proteins are common in gut dysfunctions, such as postweaning diarrhea, reflux esophagitis, irritable bowel syndrome, and IBD [36]. For example, TJ proteins $\mathrm{ZO}-1$ and occludin were decreased in irritable bowel syndrome [37, 38]. Recent studies have revealed that changes in TJ proteins could be potential biomarkers and targets for treatment, of these intestinal conditions $[14,19,39]$. Antibiotics, on the other hand, have little beneficial effect on disruptions of TJ proteins in intestinal diseases. Given the effectiveness of $L$. reuteri LR1 on TJ proteins demonstrated here, it might provide a safe and efficacious treatment strategy for many intestinal disorders caused by damage to the epithelial barrier.

In conclusion, $L$. reuteri LR1, in a MLCK-dependent manner, offset the decreased content of TJ proteins ZO-1 and occludin in IPEC-1 cells, otherwise caused by the damaging effect of ETEC K88 infection, suggesting that L. reuteri LR1 might offer promise for treating intestinal disorders with impaired function of the epithelial barrier.

\section{Data Availability}

The data used to support the findings of this study are available from the corresponding author upon request.

\section{Disclosure}

The abstract of this manuscript was presented as a conference paper for the 2017 ASAS-CSAS Annual Meeting and Trade Show in Baltimore, MD (https://www.asas. org/docs/default-source/annual/2017/abstractsannual2017_ web.pdf?sfvrsn=d81a46d1_2).

\section{Conflicts of Interest}

The authors declare no competing financial interests.

\section{Acknowledgments}

This study was funded by the National Natural Science Foundation of China (Grant no.38201815), Science and Technology Planning Project of Guangzhou (201607020035), the Special Fund for Agro-scientific Research in the Public Interest (no. 201403047), China Agriculture Research System (CARS-35), the Special Foundation of President of the Guangdong Academy of Agricultural Sciences (201710), and Science and Technology Planning Project of Guangdong Province (2016A020210041 and 2018A030310191).

\section{References}

[1] A. Farhadi, A. Banan, J. Fields, and A. Keshavarzian, "Intestinal barrier: an interface between health and disease," Journal of Gastroenterology and Hepatology, vol. 18, no. 5, pp. 479-497, 2003.

[2] J. Landy, E. Ronde, N. English et al., "Tight junctions in inflammatory bowel diseases and inflammatory bowel disease associated colorectal cancer," World Journal of Gastroenterology, vol. 22, no. 11, pp. 3117-3126, 2016.

[3] R. Y. Lu, W. X. Yang, and Y. J. Hu, "The role of epithelial tight junctions involved in pathogen infections," Molecular Biology Reports, vol. 41, no. 10, pp. 6591-6610, 2014.

[4] H. Wang, C. Zhang, G. Wu et al., "Glutamine enhances tight junction protein expression and modulates corticotropinreleasing factor signaling in the jejunum of weanling piglets," Journal of Nutrition, vol. 145, no. 1, pp. 25-31, 2015.

[5] S. Ludidi, D. Jonkers, E. Elamin et al., "The intestinal barrier in irritable bowel syndrome: subtype-specific effects of the systemic compartment in an in vitro model," PLoS One, vol. 10, no. 5, article e0123498, 2015.

[6] P. Dietrich and C. Hellerbrand, "Non-alcoholic fatty liver disease, obesity and the metabolic syndrome," Best Practice \& Research. Clinical Gastroenterology, vol. 28, no. 4, pp. 637653, 2014.

[7] F. Han, H. Zhang, X. Xia et al., "Porcine $\beta$-defensin 2 attenuates inflammation and mucosal lesions in dextran sodium sulfate-induced colitis," Journal of Immunology, vol. 194, no. 4, pp. 1882-1893, 2015.

[8] R. Mennigen, K. Nolte, E. Rijcken et al., "Probiotic mixture VSL\#3 protects the epithelial barrier by maintaining tight junction protein expression and preventing apoptosis in a murine model of colitis," American Journal of PhysiologyGastrointestinal and Liver Physiology, vol. 296, no. 5, pp. G1140-G1149, 2009.

[9] I. Cho, S. Yamanishi, L. Cox et al., "Antibiotics in early life alter the murine colonic microbiome and adiposity," Nature, vol. 488, no. 7413, pp. 621-626, 2012.

[10] M. Wlodarska, B. Willing, K. M. Keeney et al., "Antibiotic treatment alters the colonic mucus layer and predisposes the host to exacerbated Citrobacter rodentium-induced colitis," Infection and Immunity, vol. 79, no. 4, pp. 1536-1545, 2011.

[11] H. Yi, L. Zhang, Z. Gan et al., "High therapeutic efficacy of cathelicidin-WA against postweaning diarrhea via inhibiting inflammation and enhancing epithelial barrier in the intestine," Scientific Reports, vol. 6, no. 1, article 25679, 2016.

[12] K. A. Eaton, A. Honkala, T. A. Auchtung, and R. A. Britton, "Probiotic Lactobacillus reuteri ameliorates disease due to enterohemorrhagic Escherichia coli in germfree mice," Infection and Immunity, vol. 79, no. 1, pp. 185-191, 2010.

[13] Y. Liu, D. Q. Tran, N. Y. Fatheree, and J. Marc Rhoads, "Lactobacillus reuteri DSM 17938 differentially modulates effector memory $\mathrm{T}$ cells and Foxp $3+$ regulatory $\mathrm{T}$ cells in a mouse model of necrotizing enterocolitis," American Journal 
of Physiology-Gastrointestinal and Liver Physiology, vol. 307, no. 2, pp. G177-G186, 2014.

[14] F. Yang, A. Wang, X. Zeng, C. Hou, H. Liu, and S. Qiao, "Lactobacillus reuteri $\mathrm{I} 5007$ modulates tight junction protein expression in IPEC-J2 cells with LPS stimulation and in newborn piglets under normal conditions," BMC Microbiology, vol. 15, no. 1, p. 32, 2015.

[15] T. L. Talarico, I. A. Casas, T. C. Chung, and W. J. Dobrogosz, "Production and isolation of reuterin, a growth inhibitor produced by Lactobacillus reuteri," Antimicrobial Agents and Chemotherapy, vol. 32, no. 12, pp. 1854-1858, 1988.

[16] J. Walter, R. A. Britton, and S. Roos, "Host-microbial symbiosis in the vertebrate gastrointestinal tract and the Lactobacillus reuteri paradigm," Proceedings of the National Academy of Sciences of the United States of America, vol. 108, Supplement 1, pp. 4645-4652, 2011.

[17] J. K. Spinler, M. Taweechotipatr, C. L. Rognerud, C. N. Ou, S. Tumwasorn, and J. Versalovic, "Human-derived probiotic Lactobacillus reuteri demonstrate antimicrobial activities targeting diverse enteric bacterial pathogens," Anaerobe, vol. 14, no. 3, pp. 166-171, 2008.

[18] Y. Liu, N. Y. Fatheree, N. Mangalat, and J. M. Rhoads, "Human-derived probiotic Lactobacillus reuteri strains differentially reduce intestinal inflammation," American Journal of Physiology-Gastrointestinal and Liver Physiology, vol. 299, no. 5, pp. G1087-G1096, 2010.

[19] Y. Liu, N. Y. Fatheree, N. Mangalat, and J. M. Rhoads, "Lactobacillus reuteristrains reduce incidence and severity of experimental necrotizing enterocolitis via modulation of TLR4 and NF- $\kappa B$ signaling in the intestine," American Journal of Physiology-Gastrointestinal and Liver Physiology, vol. 302, no. 6, pp. G608-G617, 2012.

[20] G. A. Preidis, D. M. Saulnier, S. E. Blutt et al., "Probiotics stimulate enterocyte migration and microbial diversity in the neonatal mouse intestine," The FASEB Journal, vol. 26, no. 5, pp. 1960-1969, 2012.

[21] S. Segawa, M. Fujiya, H. Konishi et al., "Probiotic-derived polyphosphate enhances the epithelial barrier function and maintains intestinal homeostasis through integrin-p 38 MAPK pathway," PLoS One, vol. 6, no. 8, article e23278, 2011.

[22] L. Shen, L. Su, and J. R. Turner, "Mechanisms and functional implications of intestinal barrier defects," Digestive Diseases, vol. 27, no. 4, pp. 443-449, 2009.

[23] L. Shen, "Tight junctions on the move: molecular mechanisms for epithelial barrier regulation," Annals of the New York Academy of Sciences, vol. 1258, no. 1, pp. 9-18, 2012.

[24] Z. Wang, L. Wang, Z. Chen et al., "In vitro evaluation of swinederived Lactobacillus reuteri: probiotic properties and effects on intestinal porcine epithelial cells challenged with enterotoxigenic Escherichia coli K88," Journal of Microbiology and Biotechnology, vol. 26, no. 6, pp. 1018-1025, 2016.

[25] Y. Gao, F. Han, X. Huang, Y. Rong, H. Yi, and Y. Wang, "Changes in gut microbial populations, intestinal morphology, expression of tight junction proteins, and cytokine production between two pig breeds after challenge with Escherichia coli K88: a comparative study," Journal of Animal Science, vol. 91, no. 12, pp. 5614-5625, 2013.

[26] K. M. Yang, Z. Y. Jiang, C. T. Zheng, L. Wang, and X. F. Yang, "Effect of Lactobacillus plantarum on diarrhea and intestinal barrier function of young piglets challenged with enterotoxigenic Escherichia coli K88," Journal of Animal Science, vol. 92, no. 4, pp. 1496-1503, 2014.
[27] H. Y. Liu, S. Roos, H. Jonsson et al., "Effects of Lactobacillus johnsonii and Lactobacillus reuteri on gut barrier function and heat shock proteins in intestinal porcine epithelial cells," Physiological Reports, vol. 3, no. 4, article e12355, 2015.

[28] W. Zhang, Y. H. Zhu, J. C. Yang, G. Y. Yang, D. Zhou, and J. F. Wang, "A selected Lactobacillus rhamnosus strain promotes EGFR-independent Akt activation in an enterotoxigenic Escherichia coli K88-infected IPEC-J2 cell model," PLoS One, vol. 10, no. 4, article e0125717, 2015.

[29] D. Ahl, H. Liu, O. Schreiber, S. Roos, M. Phillipson, and L. Holm, "Lactobacillus reuteri increases mucus thickness and ameliorates dextran sulphate sodium-induced colitis in mice," Acta Physiologica, vol. 217, no. 4, pp. 300-310, 2016.

[30] Y. Wu, C. Zhu, Z. Chen et al., "Protective effects of Lactobacillus plantarum on epithelial barrier disruption caused by enterotoxigenic Escherichia coli in intestinal porcine epithelial cells," Veterinary Immunology and Immunopathology, vol. 172, pp. 55-63, 2016.

[31] J. Berkes, V. K. Viswanathan, S. D. Savkovic, and G. Hecht, "Intestinal epithelial responses to enteric pathogens: effects on the tight junction barrier, ion transport, and inflammation," Gut, vol. 52, no. 3, pp. 439-451, 2003.

[32] S. Resta-Lenert and K. E. Barrett, "Live probiotics protect intestinal epithelial cells from the effects of infection with enteroinvasive Escherichia coli (EIEC)," Gut, vol. 52, no. 7, pp. 988-997, 2003.

[33] N. V. Bogatcheva, M. A. Zemskova, C. Poirier et al., "The suppression of myosin light chain (MLC) phosphorylation during the response to lipopolysaccharide (LPS): beneficial or detrimental to endothelial barrier?," Journal of Cellular Physiology, vol. 226, no. 12, pp. 3132-3146, 2011.

[34] D. Yu, A. M. Marchiando, C. R. Weber et al., "MLCK-dependent exchange and actin binding region-dependent anchoring of ZO-1 regulate tight junction barrier function," Proceedings of the National Academy of Sciences of the United States of America, vol. 107, no. 18, pp. 8237-8241, 2010.

[35] X. Cheng, X. Wang, Y. Wan, Q. Zhou, H. Zhu, and Y. Wang, "Myosin light chain kinase inhibitor ML7 improves vascular endothelial dysfunction via tight junction regulation in a rabbit model of atherosclerosis," Molecular Medicine Reports, vol. 12, no. 3, pp. 4109-4116, 2015.

[36] T. Oshima and H. Miwa, "Gastrointestinal mucosal barrier function and diseases," Journal of Gastroenterology, vol. 51, no. 8, pp. 768-778, 2016.

[37] M. Coëffier, R. Gloro, N. Boukhettala et al., "Increased proteasome-mediated degradation of occludin in irritable bowel syndrome," The American Journal of Gastroenterology, vol. 105, no. 5, pp. 1181-1188, 2010.

[38] N. Bertiaux-Vandaële, S. B. Youmba, L. Belmonte et al., "The expression and the cellular distribution of the tight junction proteins are altered in irritable bowel syndrome patients with differences according to the disease subtype," The American Journal of Gastroenterology, vol. 106, no. 12, pp. 2165-2173, 2011.

[39] J. Sun, Y. Qiao, C. Qi et al., "High-fat-diet-induced obesity is associated with decreased antiinflammatory Lactobacillus reuteri sensitive to oxidative stress in mouse Peyer's patches," Nutrition, vol. 32, no. 2, pp. 265-272, 2016. 


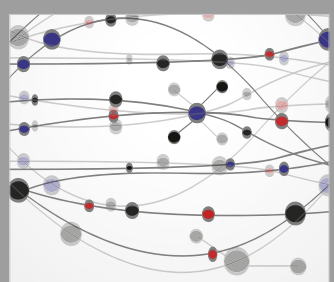

The Scientific World Journal
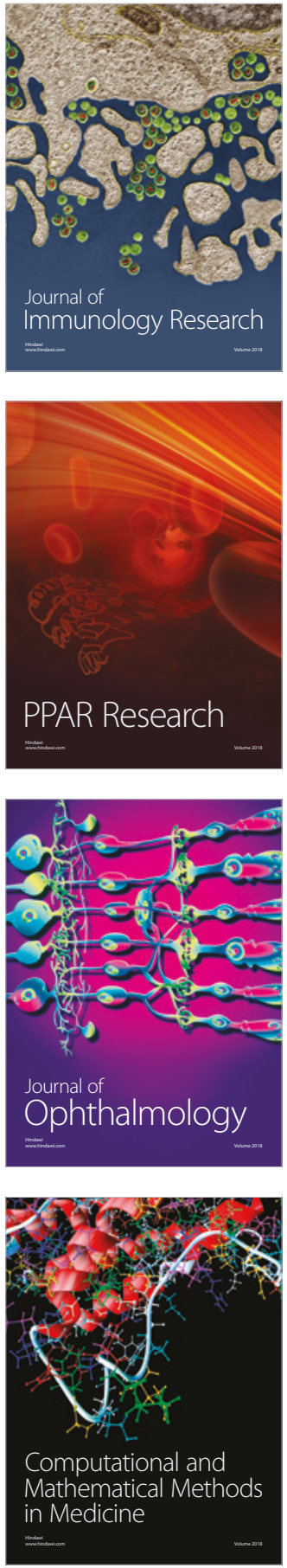

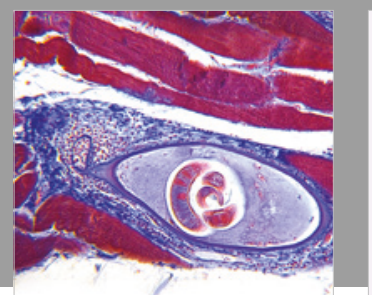

Gastroenterology Research and Practice

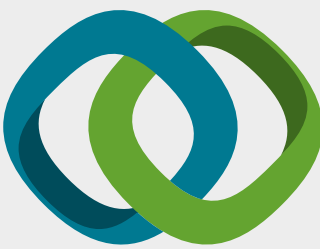

\section{Hindawi}

Submit your manuscripts at

www.hindawi.com
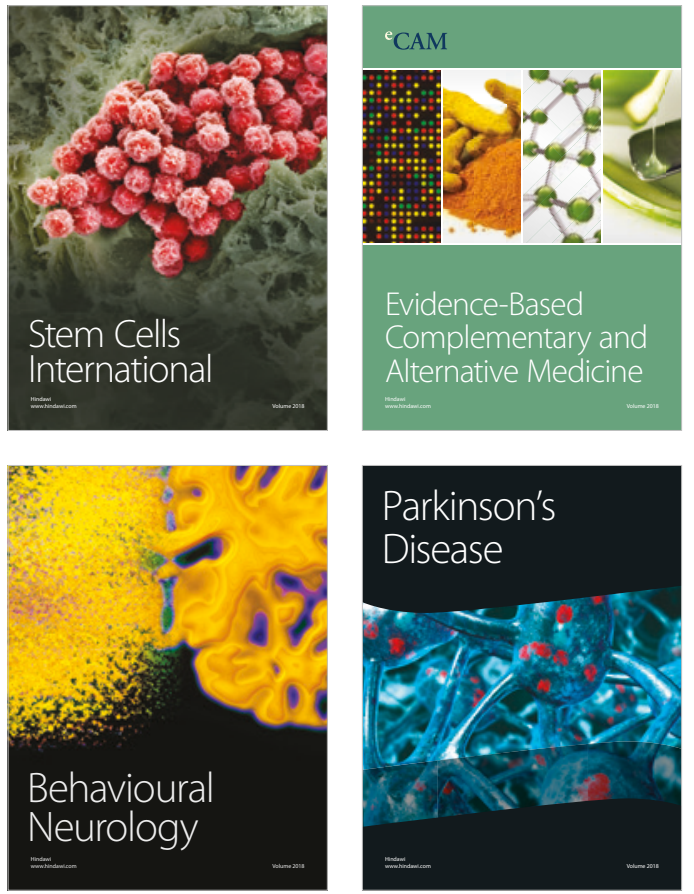

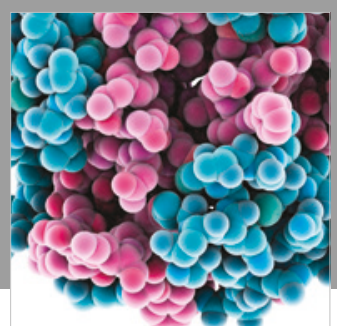

ournal of

Diabetes Research

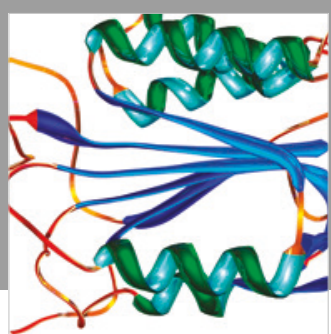

Disease Markers
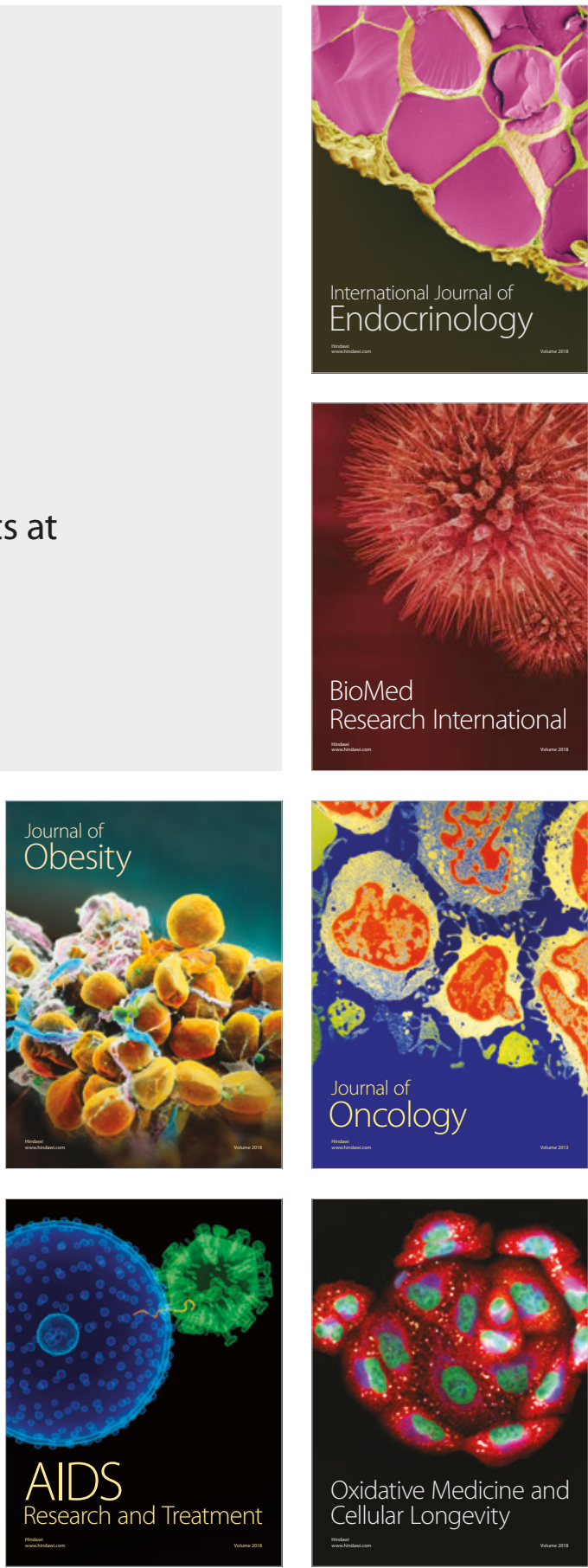